\title{
Construction of Blended Learning Model Based on Reciprocal Peer Tutoring XIONG Su-juan
}

\author{
Ningbo City College of Vocational Technology, Yinzhou District, Ningbo, Zhejiang, China
}

\begin{abstract}
Key words: Reciprocal Peer Tutoring; Blended Interactive Learning Model; In-class Learning; Online Learning; Advantage
\end{abstract}

\begin{abstract}
With the rapid development of modern education technology, the blended learning mode has attracted more and more attention from the scholars at home or abroad. On the basis of Reciprocal Peer Teaching, constructing the blended interactive learning model can make full use of the advantages of both in-class learning and online learning to promote students' autonomous learning ability, and fully implement the student-centered concept in the teaching practice. Additionally, it also helps to solve the problem of "broadcast-based" teaching in the large-size class, improve the students' digital learning quality, and enhance students' ability to solve learning problems so as to ensure the improvement of the teaching quality.
\end{abstract}

\section{基于 “同伴导学制” 的混合式互动学习模式构建}

\author{
熊素娟

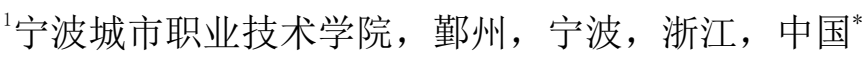

关键词: 同伴导学制; 混合式互动学习模式; 课堂学习; 网络在线学习; 优势互补

摘要：随着现代教学技术的快速发展, 混合式学习模式越来越受到教育界的普遍关注。通过 构建基于 “同伴导学制” 的混合式互动学习模式, 可以充分利用课堂学习和网络在线学习的 优势互补来促进学生自主学习能力的提高, 体现以学生为主体, 教师为主导的教学理念, 解 决大班 “广播式” 教学现状, 提升学生数字化学习的素养, 增强学生解决学习问题的能力, 从而提高教学质量。

\section{1.引言}

混合式互动学习聚焦于把传统学习方式的优势和网络化学习的优势结合起来, 这样既能 发挥教师引导、启发、监控教学过程的主导作用, 同时也可以充分体现学生作为学习过程主 体的主动性、积极性与创造性（何克抗, 2005)。[1] 在混合式学习过程中, 教师既可以和学 生进行面对面的传统教学, 又可以通过网络平台进行在线教学。另外, 教师还可以在网络平 台进行备课、设计教学活动、查看学习情况、检查学生作业完成情况等, 而学生除了在课堂 中进行学习之外, 还可以在线上开展自主学习和同伴互助学习, 顺利完成学习任务。教学实 践表明, 基于“同伴导学制”课堂教学活动能够保证混合式学习模式实施的有效性, “同伴导学 制”混合式互动学习模式是一种行之有效的教学模式。

同伴导学制 (Reciprocal peer tutoring) 是由美国学者John Fantuzzo和他的同事最早提出的, 是一种基于小组为单位开展的同伴学习活动，小组内所有成员轮流做“指导者”和“被指导者”, 实现小组成员间的互助学习、互相支持、互相评价。[2] [3]近年来，国内外同行对于“同伴导学” 
的研究越来越深入, 大量研究都表明同伴互教或者同伴辅导可以有效提高学生的学习能力, 无论是导学者还是接受导学者都认为在此过程中他们收获甚多 (Slavin, 1991; Rittschof \& Griffin,2001）。[4] [5]但是也有学者指出, 同伴互教的内容多为接受性的基础知识, 如果同伴 的学习能力不强, 或者对知识的理解正确性不高, 会对同伴互助学习的效果产生消极的影响。 因此, 我们在混合式学习模式下开展“同伴导学制”的教学实践经验表明, 在开展同伴导学活 动前教师要帮助学生做好知识的储备, 要求学生课前自主学习教师在网络学习平台上上传的 视频学习材料、文本导学教案, 同时在网络学习平台上进行疑难问题的讨论学习; 在传统的 课堂教学中, 根据教师给出的任务单, 组建课堂学习小组, 开展课内导学学习活动。

\section{2.同伴导学制的理论基础}

同伴导学制深层次的内涵融合了合作学习理论、学习金字塔理论、群体动力理论等不同 教学理论。

\section{1合作学习理论}

有关合作学习（Cooperative Learning）的正式研究始于20世纪50年代的美国，一经提出 就引起了西方国家的广泛重视和研究, 并形成了多种合作学习模式。[6]作为合作学习的主要 代表人物, 以色列特拉维夫大学沙伦博士对合作学习的内涵进行了界定: “合作学习是组织和 促进课堂教学的一系列方法的总称。学生之间在学习过程中的合作则是所有这些方法的基本 特征。在课堂上, 同伴之间的合作是通过组织学生在小组活动中实现的, 小组通常由 $3-5$ 人组 成, 小组成员间相互交流展开学习”。“同伴导学制”正是合作学习的一种典型形式, 凸显成员 间的相互支持、相互交流完成共同的任务，实现学习目标。

\section{2学习金字塔理论}

学习金字塔理论是由美国学者埃德加·戴尔 (Edgar Dale) 在1946年提出的。此后, 美国 缅因州National Training Laboratories也做过类似的研究, 称之为“Learning Pyramid”, 其结论与 埃德加·戴尔的结论相似。本文引用的是美国缅因州国际训练实验室研究的金字塔理论（如图 1所示）。[7][8]学习金字塔理论表明: 学习者采用不同的学习方法, 获得的学习效果不同, 对 知识的保持率差异较大。学习方法可以分为被动学习型和主动学习型。被动学习 (听讲、阅 读、试听、演示) 的学习留存率较低，从5\%-30\%不等; 而主动学习（讨论、实践、教授给别 人) 的学习留存率较高, 从 $50 \%-90 \%$ 不等。其中在所有的学习方式中, 向别人讲授相互教这 种学习方式的知识留存率最高。这一理论也为“同伴导学制”的实施提供了有力的理论基础。

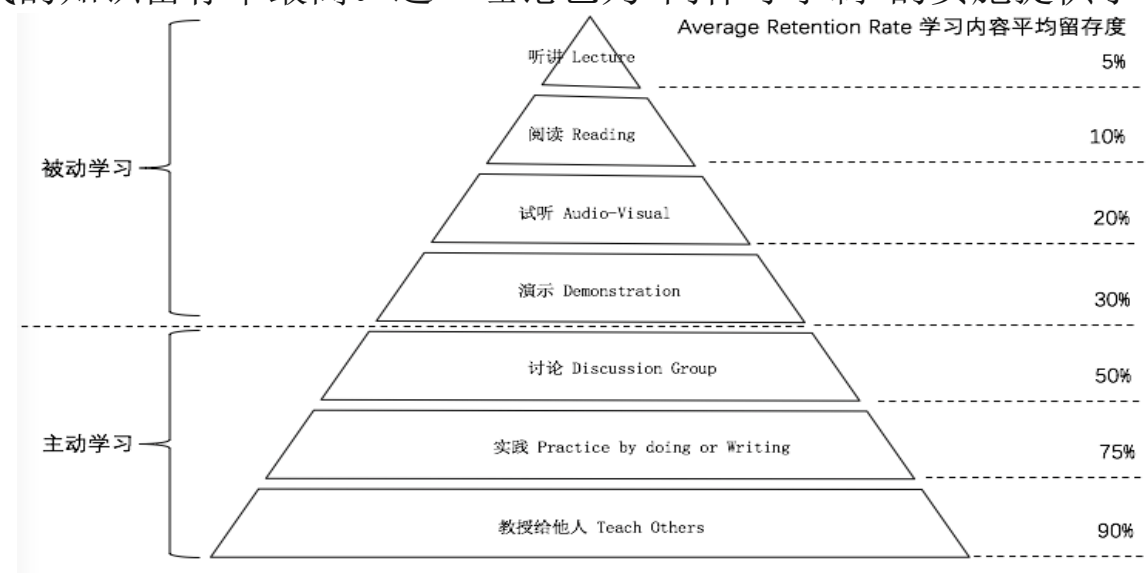

图1:Learning Pyramid （学习金字塔）

来源: National Training Laboratories. Bethel, Maine

(美国缅因州的国家训练实验室) 


\section{3群体动力理论}

群体动力理论是由美籍德国人库乐特勒温在1939年首次提出。他指出团体中的各种潜在 的动力间具有交互作用, 团体会对个体行为产生一定的影响, 团体成员之间具有相互的依存 关系, 尤其团体成员为了团体间的成就会去做创新的探索。[9] [10]群体动力理论表明在小组活 动中, 小组成员与小组整体具有相互的依存关系, 会为小组的集体荣誉贡献自己的力量, 发 挥自己最大的潜能。

\section{3.基于同伴导学的混合式互动学习模式的构建}

基于同伴导学的混合式互动学习模式的构建, 主要涉及课前自主学习阶段、课内探索学 习阶段以及面授后的梳理总结阶段，模式如图2所示。

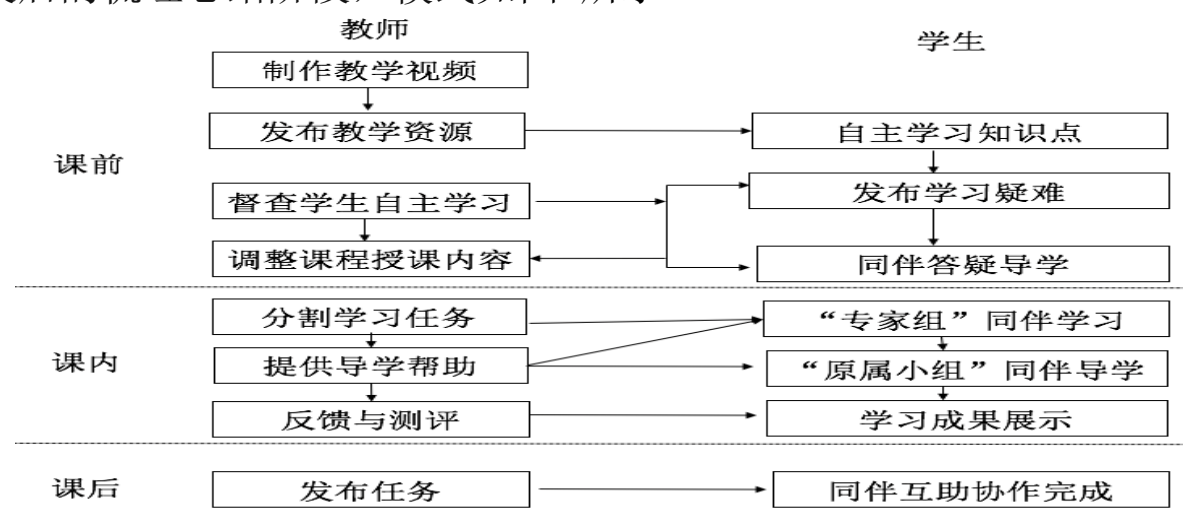

图2：基于“同伴导学制”的混合式互动学习模式

这一模式充分体现了混合式学习、合作学习、学习金字塔理论和群体动力理论的内涵。 课前, 学生借助移动教学平台, 采用自主学习和同伴互助导学的方式开展自主学习、合作学 习。课中, 学生在开展小组合作式的探究学习基础上, 将探究的知识讲授给原属小组成员, 这正体现了学习金字塔中提及到的主动学习, 凭借讨论、教授给别人的方式可以固化学习内 容, 知识留存率较高。同时, 教师在发放检测题目时采用以小组为单位的方式, 可以有效激 发学生学习热情, 充分调动小组合作学习、自主学习的原生动力, 从而提升小组的内聚力。

\section{1基于同伴导学的混合式互动学习模式开展的准备活动}

1、原属学习小组组建。教师可以通过测试、问卷、访谈的方式了解学生的学习基础、性 格特点、性别差异、学习能力、信息化水平程度, 并依据 “同组异质, 异组同质”的分组原则 对学生进行分组。每组成员具有不同的学习层次、不同的性格特征, 即同组异质; 同时每个 小组成员的异质，使得全班各小组间具有同质性，有利于小组间的交流和竞争。通常情况下， 一个小组的成员数在 6 人左右。

2、混合式学习策略培训。开展自主学习前, 教师首先要对学生开展混合式学习所需要的 自主学习策略、网络学习平台操作方式、混合式授课模式、课程考核方案等进行有序的梳理, 并以视频资源或文本资源的形式上传至学习平台, 供学生了解学习。

\section{2基于同伴导学的混合式学习模式实施步骤}

1、课前自主学习阶段。在同伴导学的混合式学习实施过程中, 我们采用了“蓝墨云”班 课移动学习平台。教师在授课前, 将学习任务单、学习材料 (视频、文件) 上传到“蓝墨云” 班课的“资源”中, 要求学生按照任务单提示完成课前自主学习。学生可以通过手机随时随地 在班课中查看学习资源, 并将学习过程中遇到的疑难问题发送在学习平台的“答疑讨论”栏中。 为了激励学生的学习积极性, 教师会汶览学生参与答疑情况, 并根据学生的学习提问情况赋 予相应的经验值奖励（各类活动的经验值按一定的权重折合计入学生的期末总评成绩）。学 生利用云班课平台进行互助学习, 对同伴提出的疑问进行讨论、解答问题。教师会对同伴答 
疑情况进行“经验值”赋分奖励, 鼓励学生的课前同伴互助学习; 同时, 根据查看学生的疑难 问题调整课堂授课内容，做到真正基于问题导向的因材施教。

2、课内探索学习阶段。基于同伴导学混合式学习模式中的课堂教学与传统的课堂教学有 很大程度上的不同。混合式教学模式的课堂教学是学生对知识的应用和提高的过程, 更加强 调学生的主动参与来实现其对知识的内化, 因此教学活动的设计应该以学生的探究学习为主 线。课堂面授活动可以分为以下步骤:

(1) 学习任务分割。在课堂面授环节, 以小组形式开展同伴导学活动。根据学生课前自 主学习提问的情况, 结合教学内容的重难点, 教师将语言学习分割成难易程度相近的学习任 务, 学习任务数与每一小组成员数相同。

例如, 教师根据教学内容, 录制了9个视频供学生课前学习。课前教师根据学生的疑问将 关于 9 个视频学习后的疑难问题分成了 6 组学习任务（授课班级原属小组成员均为 6 人），6组 学习任务分别命名为 $A 、 B 、 C 、 D 、 E 、 F 、 G 。$

(2) 组建“专家组”同伴学习。教师要求每一组“原属小组”的每一位成员在教师给定的学 习任务中任意选取一组。“原属小组”中选取同一组学习任务的同学重新组成一个小组, 称之 为“专家组”。例如, 在笔者授课的班级中, 来自6个“原属小组”中选择任务A的同学重新组建 一个新的小组, 命名为“专家组 $A$ ”。“专家组A”的所有成员就自己选择的任务A进行研讨式、 理解性学习。之后, 每位“专家组”成员负责回到“原属小组”担任所选择的话题内容的“主讲”。 因此, 在“专家组”讨论过程中, 每位成员必须认真参与讨论、解疑、记录问题的解决方式。 在此过程中, 教师在各组之间进行巡视, 提供必要的帮助和指导, 确保学生探究学习的有效 性。

（3）回归“原属小组”同伴导学。“专家组”成员在进行研讨式学习后，各成员回到自己的 “原属小组”进行同伴导学，即各成员轮流充当“教师”的角色，在小组内将自己在“专家组”的 学习成果清晰地传授给其他同学。此时, 教师也需要巡视学生的导学过程中, 记录学生在导 学中出现的问题。

(4) 反馈与测评。教师针对学生导学中出现的问题进行解疑答惑, 帮助学生梳理学习内 容。为了固化学生所学知识, 教师通过蓝墨云班课, 发布测试题目, 题目可分别设计为个体 记分和小组记分形式, 既可以了解学生个体的学习情况, 也可以用来激励小组的团队合作能 力。正如群体动力理论指出的, 团体中的各种潜在的动力会促使团体成员积极投入新的创新, 并能因为小组的集体荣誉感而发挥自己所有的潜能。因此, 在反馈和检测阶段设计小组测试 题目，可以有效地激发小组成员的综合潜能，高效发挥成员个体的最大潜能。

3、课后梳理总结阶段。教师发放课后导学案引导学生对课前、课中的学习活动进行回顾, 帮助学生完善知识结构。教师要求学生在提交个人作业前, 同伴间需对相互的作业进行评价 点评, 这样可以使学生在评价作业时开展交流和合作, 引导学生积极参与, 增进学生间的多 向交流, 从而利于学生取长补短, 让学生进一步在与同伴学习的比较中认识自我。

\section{3基于同伴导学的混合式学习模式的教学评价}

基于同伴导学的混合式学习模式的教学评价分为三个维度, 即在线学习平台提供的学生 学习评价、小组成员间的匿名评价、教师评价。在线学习平台能将学生在学习平台上的学习 轨迹记录在案, 并能及时向教师反馈学生的自主学习问题, 以便教师及时对学生的自主学习、 合作学习情况进行督学。利用学习平台的同伴互评功能, 参与合作学习的小组成员可以采用 匿名的方式就课前疑问讨论的参与度、贡献度、课上讨论、讲解的积极性和准确性等方面进 行相互评价, 便于教师多维度收集学生的课前自主学习情况。此外, 教师根据课中知识检测 环节中学生的学习表现对学生进行量化评价。 


\section{4.基于同伴导学制混合式学习模式的优势}

同伴导学混合式学习模式是对传统填鸭式、启发式教学模式的挑战, 是对课堂主客地位 的一种颠覆, 是高科技进课堂与时俱进的一种学习策略。归纳起来主要有如下优势:

\section{1实现在线学习和传统课堂教学的优势互补}

在线学习不受时间、空间、地域的限制, 学生可以利用碎片化的时间自主安排学习。此 外, 利用自主学习平台, 可以及时得到同伴、教师的帮助, 在一定程度上提高了学生的学习 效率。在教育技术的不断推动下, 传统的面授形式也发生了改变, 不再是教师单纯的知识灌 输, 教师根据学生课前学习的难点设计教学活动, 根据课堂教学中学生的学习状态实时调整 教学策略、教学活动。通过线上学生自学、同伴导学, 线下小组探究、同伴教学的方式充分 发挥在线学习和传统课堂教学的优势互补。

\section{2充分体现以学生为主体, 教师为主导的教学理念}

基于同伴导学的混合式教学模式的开展, 可以有效增强学生学习的主动性, 改变了学生 过去“被动学习”的模式。而教学过程也不是单纯的知识传授过程, 更注重学生知识生成过程。 教师通过利用多媒体资源、网络资源、学习平台给学生的自主学习提供资源和必要的帮助, 从而真正地发现学生的学习需求, 鼓励学生自主探究与合作交流。新的教学中强调学生自主 探究, 通过交流与合作去寻找解决问题的方法, 感悟知识的生成过程。而随着 “知识传授”逐 渐向“帮助学生”转移, 教师的角色也将演化为“协助者”、“指导者”。同伴导学制的教学理念 是充分以学生为主体, 教师为主导。改变了传统的教学模式, 取而代之的是在教师的导学下, 学生开展课前知识的自主习得, 将知识点的学习前移到课前, 自求不得时可以求助于老师, 真正将课堂还给学生, 让学生开展研究性学习, 实现知识点的固化和延伸。

\section{3解决大班 “广播式”教学现状}

传统教学中, 由于自然班级的班容量大, 教师无法实现因材施教, 授课中多采用“广播式” 教学模式。采用基于同伴导学制的混合式教学模式, 学生课前通过线上自主学习, 将个性化 的问题发布至学习平台, 同伴间答疑解惑; 课中教师组织学生以小组形式对学生提出的疑难 问题进行合作学习, 采用学与教相结合的模式实现对知识的深化学习, 固化知识技能, 关注 学生的个性化学习需求, 基于问题解决为导向开展教学, 有利于解决大班教学中“满堂灌”、“教 师一言堂”的现状。

\section{4提升学生的数字素养}

在互联网与移动互联网的技术背景下, 提升学生的数字素养成为教育界热议的主题。数 字素养的提升不仅需要学生具有基本的技术技能, 而且需要具备一些行为技能, 如协作能力 和批判性思维能力。同伴导学的混合式教学模式实施过程中, 学生在自主学习时要求能熟练 应用数字化平台, 有效利用数字化资源; 在同伴互助学习时, 相互学习, 学会搜索、甄别、 处理各种信息; 针对同伴提出的疑问进行答疑时, 学生的思维会不断拓展, 批判性思维不断 增强, 在很大程度上提高了学生的数字素养的行为技能。

\section{5.结语}

随着教育信息化技术的不断发展，未来的课堂一定不是单向的、无互动的课堂。基于同 伴导学制的混合式教学是多向的、互动的过程, 既包括师生之间的互动, 更包含生生之间的 持续互动。通过同伴导学中的“互教” 模式, 激发了学生参与小组合作学习的热情, 加强了学 生解决问题的能力, 固化了学生的知识, 有助于学生批判性思维的形成。基于同伴导学制的 混合式教学使得师生之间的交流变得更加顺畅, 师生角色翻转, 教师的“教”变为“导”, 学生 
单纯的“学”变为“学-教”。通过建立“专家组”课中问题研讨的形式, 具有不同学习能力的学生 合作学习, 共同解决问题, 给学生提供发表观点的“安全感”, 鼓励学生大胆表达、积极思考。 此外，“专家组”成员在讨论结束后回归“原属小组”进行知识的传输, 这一模式也有利于提高 学生的表达能力、提升学生的自信心以及责任意识。而在面授环节的知识检测与反馈环节, 充分体现了教师“导学”的作用, 针对学生的知识、能力的欠缺之处进行及时地了解、补充与 提升。基于同伴导学的混合式学习模式的构成成为一个产生问题、解决问题、固化知识、迁 移知识和创新知识的过程。

\section{致谢}

基金项目：2017 年度全国教育信息技术研究课题“基于 CIPP 评估模式的慕课评价体系构建与 应用研究”，（项目编号：176140007） 2017 年度宁波城市职业技术学院校级慕课建设项 目“基础英语综合 1 ”

\section{References}

[1] He Kekang. New Development of Educational Technology from the Viewpoint of Blending Learning[J]. Journal of National Academy of Education Administration,2005(9):38-48.

[2] Fantuzzo, J.W., Dimeff, L.A., \& Fox, S.L. Reciprocal Peer Tutoring: a multimodal assessment of effectiveness with college students[J]. Teaching of Psychology, 1989a (16):133-135.

[3] Wolfe, J.A., Fantuzzo, J.W., \& Wolfe, P.K.The effects of reciprocal peer management and group contingencies on the arithmetic proficiency of underachieving students[J]. Behavior Therapy,1986 (17): 253-265.

[4] Rittschof, Kent A.\& Griffin, Bryan W. Reciprocal Peer Tutoring: re-examining the value of a co-operative learning technique to college students and instructors[J]. Educational Psychology, 2001(03):313-330.

[5] Slavin,R.E. Synthesis of research on cooperative learning[J]. Educational Leadership, 1991(48):71-82.

[6] Wang Tan. Cooperative Learning : Theory and Practice [M].China Human Resources \&Social Security Publishing Group, 2002.

[7] Dale E. Audio-Visual Methods in Teaching. 3rd Ed. New York: Holt, Rinehart \& Winston; 1969:108.

[8] Jiang Yanling \& Xu Tong. Learning Pyramid Theory in the Application and Practice of Flipped Classroom[J]. China Educational Technology,2014(07):133-138.

[9] Forsyth, D.R. Group dynamics. Belmont, CA: Wadsworth, Cengage Learning;2010.

[10]Wang Jianxin. Cooperative Learning of English from Perspective of Group Dynamic Theory[J]. China Educational Technology \& Equipment,2009(30):36-37. 